LA-10811-MS

UC-15

Iseured: Fobruary 1987

LA--108II-MS

DE87 004767

\title{
Fundamental Data Analyses for Measurement Control
}

\author{
K. Campbell \\ G. L. Barlich \\ B. Fazal
}

R. B. Strittmatter

\section{DISCLAIMER}

This report was prepared as an account of work sponsored by an agency of the United States Government. Neither ine United States Government nor any agency thereof, nor any of their employees, makes any warranty, express or implied, or assumes any legal liability or responsibility for the accuracy, completeness, ar usefulness of any information, apparatus, product, or process disclosed, or represents that its use would not infringe privately owned rights. Reference herein to any specific commercial product, process, or service by trade name, trademark, manufacturer, or otherwise does not necessarily constitute or imply its endorsement. recommendation, or favoring by the United States Government or any agency thereof. The views and opinions of authors expressed herein do not necessarily state or reflect those of the United States Government or any agency thereof.

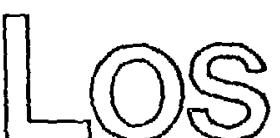

A
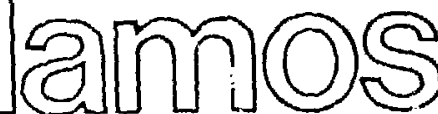


\title{
FUNDAMENTAL DATA ANALYSES FOR MRASUREMRNT CONTROL
}

\author{
by
}

K. Campbe11, G. L. Barlich, B. Fazal, and R. B. Strittmatter

\begin{abstract}
A set of measurement control data analyses was selected for use by analysts responsible for maintaining measurement quality of nuclear materials accounting instrumentation. The analyses consist of control charts for bias and precision and statistical tests used as analytic supplements to the control charts. They provide the desired detection sensitivity and yet can be interpreted locally, quickly, and easily. The control charts provide for visual inspection of data and enable an alert reviewer to spot problems possibly before statistical tests detect them. The statistical tests are useful for automating the detection of departures from the controlled state or from the underlying assumptions (such as normality).
\end{abstract}

\section{INTRODUCTION}

An important aspect of a complete measurement control program is the analysis of data from periodic control measurements of known standards. A set of measurement control data analyses has been selected to provide the desired detection sensitivity and still be easy to interpret. These criteria allow the analyst responsible for maintaining measurement quality to make local and timely review of control data. The analyses consist of control charts for bias and precision and statistical tests used as analytic supplements to the control charts. The charts allow visual inspection of data and enable an alert reviewer to spot problems possibly before they are detected by statistical tests. The statistical tests are used for automating the detection of departures from the controlled state or from the underlying assumptions, such as normality. 
The algorithms described here are

- control charts for bias,

- control charts for precision,

- sequential tests for shifts in the mean, and

- tests for randomness and normality.

A detailed review of the statistical algorithms is presented in Sec. II.

Page's sequential test, used to automate the detection of changes in bias, can signal a single observation deviating greatly from the mean or a sequence of observations deviating slightly from the mean. This test can be described as a cumulative sum (cusum) of the past observations, with a restart mechanism that prevents a recent problem from being obscured by a long history of satisfactory behavior.

Control charts and other statistical tests are based on the assumption that measurements from the control program are approximately normally distributed. Failure of normality can affect the false-alarm rate of the tests and decrease their usefulness for detecting real problems. The recommended test for normality is the Shapiro-Wilks test, applied to predetermined sample sizes.

Although randomness is not precisely defined, features exist that would not be considered random, such as extreme observations, slow upward or downward drifts, and cycles with a definite period. Detection of such nonrandom behavior would alert the analyst to look for factors affecting the measurements that could be controlled. The test based on the von Neumann ratio is sensitive to many types of nonrandom fluctuations.

The Los Alamos Safeguards Systems Group has implemented the described algorithms in a measurement control code for personal computers. The code, Measurement Control Charts and Tests (MCCAT), is being evaluated at the Cak Ridge Y-12 facility for highly enriched uranium scrap recovery.

\section{BASIC ALGORITHMS}

This section describes standard control charts and algorithms in the context of what we will call the "standard case," namely, daily control measurements of a known, constant standard where the variance of the measurement process is also assumed known. This section contains the basic 
information that will be needed by any user of the MCCAT software. Sections III and IV contain information that will probably be needed only by individuals responsible for setting up and maintaining the system.

\section{A. Introduction and Terminology}

The algorithms described are designed to be used with data collected as part of an ongoing measurement control program. Typically, one control measurement of a prescribed standard is made each day or after a fixed number of measurements on a given instrument. Archived with the result of this control measurement may be the certified value of the standard, a computed (that is, propagated) measurement error, and other relevant information, numerical or alphanumeric. Possible modifications of this scenario, including repeated measurements of the same standard or the use of two standards (to check different ranges of the instrument), do not significantly affect the procedures described here.

A sequence of control measurements made as above is referred to here as (a realization of) the "measurement process." Individual measurements (also called observations) typically cluster around an average value (or mean value) with a spread reflecting uncontrollable factors, such as a counting error in the measurement of radioactive material, small variations in atmospheric conditions and temperature, or slight differences in the procedures used by different operators. Generally, the distribution of observations about their mean value is adequately described by a Gaussian, or normal, probability distribution. It is also assumed that the result of one measurement does not in any way affect the result of another (that is, the observations are independent).

The objective of examining the archived sequence of control measurements is to determine whether the instrument (and the associated procedures and operators) continues to function at an acceptable level of precision and accuracy. If so, we say that the measurement process is "in control." Specifically, giver: a sequence of control measurements, we assume that the process continues in control as long as the average value and standard deviation of the observations do not deviate significantly (in a statistical sense) from their historical values. (Adjustments for a decaying radioactive standard or a predictably deteriorating precision are considered in Sec. III.) The algorithms described below are designed (1) to 
produce graphical displays that provide visual indications of deviation from the historical norms and (2) to provide timely, automatic indicators of such deviations, based on statistical tests of the null hypothesis that the measurement process is, in fact, in control.

\section{B. The Standard Case: Rnown Mean and Standard Deviation}

In the standard case, the measurement process is considered to be in control if the observations are independently normally distributed with known mean and standard deviation. Verification of normality and independence are the subjects of Secs. IV.C and IV.D, and these properties are assumed in all other sections. Here we are dealing with the problem of detecting deviations in the measurement process from the known mean value (that is, detecting bias) and also with changes in the historically determined standard deviation that provides a measure of the significance of differences between the actual observations and the known mean value.

The mean value is assumed to be known with high precision (without error for practical purposes). This is the situation, for example, when a certified standard is used (such as a National Bureau of Standards calibrated weight), when a supplementary measurement of the standard has been made by another more accurate method (for example, chemical analysis), or when a carefil measurement of a secondary standard has been made after the instrument has been calibrated using primary standards.

The standard deviation of the process, on the other hand, can be determined only by analysis of historical data or of data from a designed experiment. These data must be collected under circumstances that accurately reflect all of the sources of random error that will affect routine accountability measurements, for example, using several operators and continuing over a reasonably extended period of time. The determination of the historical standard deviation is discussed in Secs. IV.A and IV. $\bar{B}$.

This historical standard deviation must be carefully distinguished from specification limits claimed by the manufacturer of the instrument or required by the user. If the latter are significantly smaller than the historical limits, there may be a problem that needs to be investigated, but it is separate from the measurement control program. The limits that are relevant for measurement control are the historical, empirical ones. 
As long as the standard deviation of the observations is not significantly different from its historical value (and no bias is apparent), t... measurement process is considered to be in (statistical) control, even if the precision or accuracy of the measurements fails to meet administrative requirements.

Controlling (or at least correcting for) bias in special nuclear material (SNM) measurements is clearly important: consistent bias in a series of accountability measurements leads quickly to the appearance of inventory differences where none actually exist (or may possibly conceal a true difference). The importance of controlling the standard deviation may be less obvious. However, comparison of observed differences with a standard deviation that is, in fact, smaller than the process is capable of maintaining may lead incorrectly to the conclusion that inventory differences exist, and comparison of the observations with a standard deviation that is too large will mask bias. Changes in either direction are possible as a result of changes in procedure or personnel; change may also become apparent if the historical variance estimate was based on too few or nonrepresentative data.

\section{Shewart Control charts}

Let $\mu$ be the known mean and $\sigma^{2}$ the known variance of the measurement process when it is in control. Let $x_{t}$ denote the control measurement of the standard made at time $t$. We will consider the standardized measurement

$$
z_{t}=\frac{\left(x_{t}-\mu\right)}{\sigma} .
$$

Under the standard assumptions discussed in Sec. II.B, the $z_{t}$ are independent realizations of a normal random variable with mean zero and unit variance. In particular, about 1 observation in 400 is expected to exceed 3 in absolute value.

A Shewart control chart is a plot of the standardized measurements $z_{t}$ vs time $t$ and includes horizontal lines at zero (the mean value of the standardized measurement) and, generally, at plus and minus three 
units (the control limits). A standardized observation that falls outside these control limits indicates bias, and some action such as recalibration of the instrument or at least review of recent measurement control data is required.

A similar chart can be used to examine group means. The standardized measurements are divided sequentially into groups of $f$ ive, and the mean of each group is calculated. This value is plotted on a chart with mean 0 and control limits at $\pm 3 / \sqrt{5}$ and $\pm 2 / \sqrt{5}$.

Shewart charts are familiar to most people and are easy to interpret. A series of measurements (up to 81 observations in the MCCAT code) plotted in this way can reveal abrormalities that may have crept into the measurement process, which are not immediately detectable out of their historical context. However, the statistical tests implicit in the choice of control and warning limits are less than optimal, especially for the detection of small shifts in the mean value (although numerous ad hoc solutions to this problem have been developed). ${ }^{1}$ Section II.D describes a two-sided Page's test that, as a modification of a sequential probability ratio test, is known to have some optimal properties in terms of the length of time (number of observations) required to detect shifts in the mean.

A Shewart control chart can also be developed for control of the standard deviation of the measurement process. This chart is based on the sample standard deviation of a sequence of individual observations of length $r=5$. Specifically, the statistic

$$
(r-1) s_{t}^{2}=\sum_{i=t-r+1}^{t}\left(z_{i}-\bar{z}\right)^{2}=\frac{\sum_{i=t-r+1}^{t}\left(x_{i}-\bar{x}\right)^{2}}{\sigma^{2}}
$$

(where $\bar{z}$ is the average of the $r$ standardized observations $z_{i}$, $i=t-r+1, \ldots, t$, and similarly $\bar{x}$ is the average of the $r$ observations $x_{i}$ ) has a chi-square distribution with $r-1$ degrees of $i$ reedom, and upper and lower control limits for the sample standard deviations $s_{t}$, computed after each $r$ observations, are constructed using the tabled percentiles of this distribution. Specifically, the mean value of $s_{t}$, defined 
by Eq. (2), is $c_{r}$, given in Table $I$ for $r$ between 2 and 12 . Upper and lower action limits may be set at $j_{r}^{+}$and $j_{r}^{-}$, and optional warning 1 imits may be set at $v_{r}^{+}$and $v_{r}^{-}$.

\section{Sequential Test for Bias}

Page's test is based on a statistic closely related to the cumulative sum of the past observations. ${ }^{2}$ It has been described as a cusum test with a restart mechanism to prevent recent problems from being obscured by a long history of satisfactory instrument behavior. It can be alarmed by a single observation deviating from the mean by a large amount $[(h+k) \sigma$ in terms of the notation given below], by a short sequence of observations deviating from the mean by a smaller amount, or by a longer sequence of observations deviating by an even smaller amount. Thus, a single test encompasses many of the modifications proposed by Roberts ${ }^{1}$ as alternatives to the simple test implied by a Shewart chart with control limits at $\mathrm{m} \pm 3 \sigma$.

Table I. Mean value, action limits, and warning limits for the sample standard deviation of $r$ standardized observations

\begin{tabular}{|c|c|c|c|c|c|}
\hline $\begin{array}{l}\text { Number of } \\
\text { Grouped } \\
\text { Observations }\end{array}$ & $\begin{array}{c}\text { Mean Value } \\
\text { of } s_{t}\end{array}$ & $\begin{array}{l}\text { Jower } \\
\text { Action } \\
\text { Limit }\end{array}$ & $\begin{array}{l}\text { Upper } \\
\text { Action } \\
\text { Limit }\end{array}$ & $\begin{array}{l}\text { Lower } \\
\text { Warning } \\
\text { Limit }\end{array}$ & $\begin{array}{l}\text { Upper } \\
\text { Warning } \\
\text { Limit }\end{array}$ \\
\hline$r$ & $c_{r}$ & $j_{\bar{r}}$ & $\mathrm{j}_{\mathrm{r}}^{+}$ & $v_{\bar{r}}$ & $v_{r}^{+}$ \\
\hline 2 & 0.798 & 0.00 & 3.29 & 0.03 & 2.24 \\
\hline 3 & 0.886 & 0.03 & 2.63 & 0.16 & 1.92 \\
\hline 4 & 0.921 & 0.09 & 2.34 & 0.27 & 1.77 \\
\hline 5 & 0.940 & 0.15 & 2.15 & 0.35 & 1.67 \\
\hline 6 & 0.952 & 0.21 & 2.03 & 0.41 & 1.60 \\
\hline 7 & 0.959 & 0.25 & 1.93 & 0.45 & 1.55 \\
\hline 8 & 0.965 & 0.29 & 1.86 & 0.49 & 1.51 \\
\hline 9 & 0.969 & 0.35 & 1.81 & 0.52 & 1.48 \\
\hline 10 & 0.973 & 0.36 & 1.76 & 0.55 & 1.45 \\
\hline 11 & 0.975 & 0.38 & 1.72 & 0.37 & 1.43 \\
\hline 12 & 0.978 & 0.41 & 1.69 & 0.59 & 1.41 \\
\hline
\end{tabular}


A two-sided Page's test for deviations in the mean of the measurement process takes the following form:

1. Select a reference value $k$ (typically about 0.5) and a decision value $h$ (typically in the range of 4 to 5 ).

2. Initialize the Page statistics: $m(0)=0$ and $M(0)=0$.

3. For $t=1,2,3, \ldots$, compute

$$
m(t)=\max \left\{0, m(t-1)+z_{t}-k\right\}
$$

and

$$
M(t)=\max \left\{0, M(t-1)-z_{t}-k\right\}
$$

(max $\{x, y\}$ denotes the larger of the two numbers $x$ and $y$ ).

4. Declare the measurement process out of control at time $t$ if either $m(t)$ or $M(t)$ exceeds $h$.

Typically $h$ and $k$ are chosen so that it will take about 10 or 12 observations to detect a shift of magnitude 1 in the mean of the standardized measurements $z_{t}$ (that is, a shift of 1 standard deviation in the mean of the raw observations $x_{t}$ ) while allowing an average run length of the process in control (that is, the average time until a false alarm) to be approximately 300 to 400 observations. ${ }^{3}$

The interesting region of the $(h, k)$ parameter space is indicated in Fig. 1. The two heavy curves bound a region in which the average run length of the process in control exceeds 300 observations and the average number of observations until detection of a shift of $1 \sigma$ is less than 12 observations. Values of $(h, k)$ chosen from the right-hand side of this region lead to tests that have longer run lengths when the process is in control, but that also take longer, on the average, to detect a shift in the mean. Shaded regions indicate where (a) the average run length of the controlled process exceeds 400 observations and (b) the average time until 


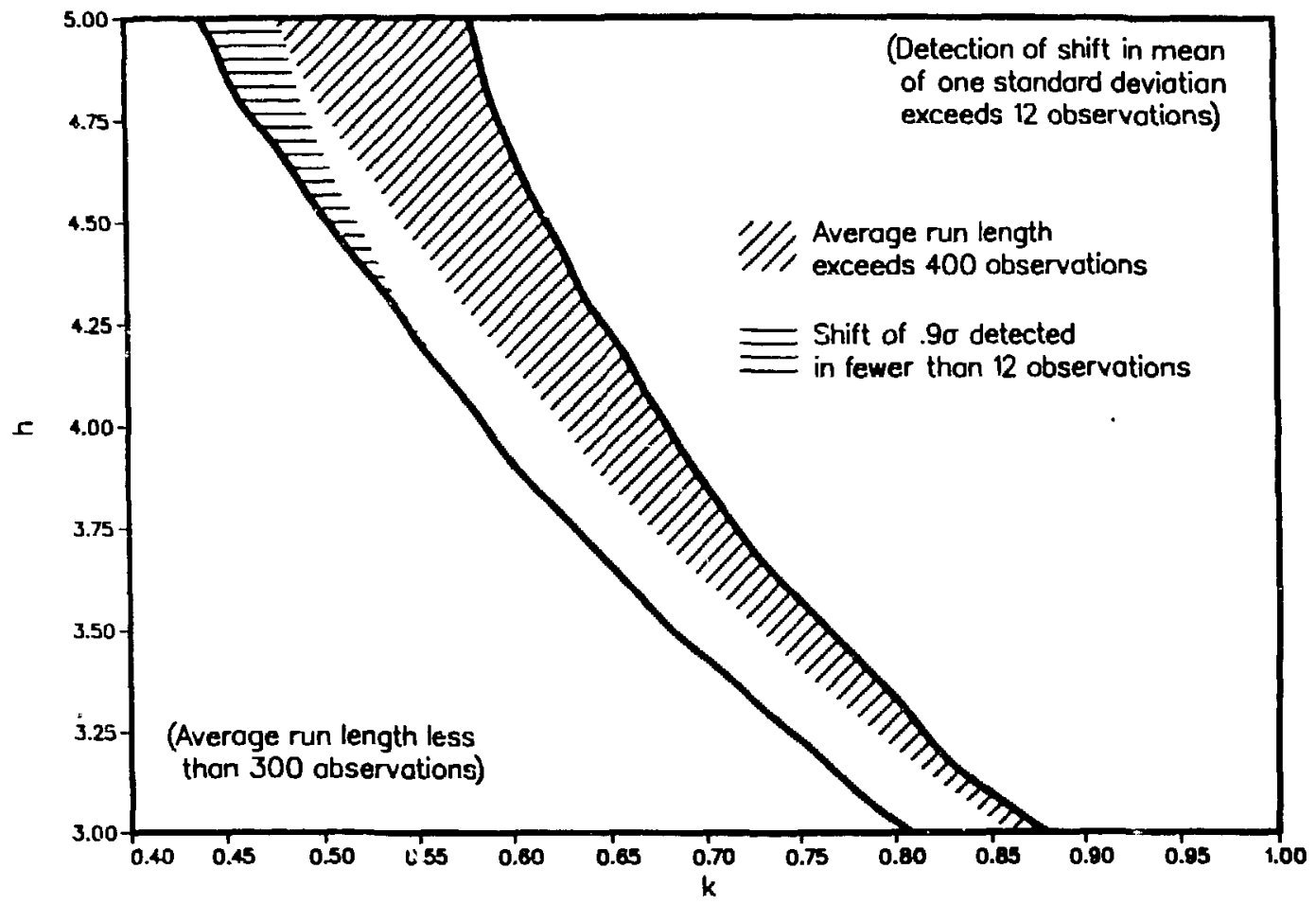

Fig. 1.

Usable region of $(h, k)$ parameter space for two-sided Page test. The two heavy curves bound a region in which the average run length of the process in control exceeds 300 observations and the average number of observations before detection of a shift of 1 standard deviation is fewer than 12. The diagonal shaded and horizontal shaded subregions correspond to longer average run lengths and to detectability of smaller shifts in 12 observations, respectively.

detection of a shift of 0.90 is less than 12 observations. From the latter we see that it is not possible to improve on the time to detection of even marginally smaller shifts without driving the average run length of the controlled process to unacceptably small values. Note also that even with an average run length of 400 observations for the process in control, a bignificani number of runs will be shorter, because the distribution of run lengths is approximately exponential; almost 1 run in 5 has fewer than 100 observations. The probability of at least one alarn occurring as a function of run length is shown in Fig. 2 for $(h, k)=(5.0,0.5)$ and Fig. 3 for $(h, k)=(7 . b, 0.5)$. 


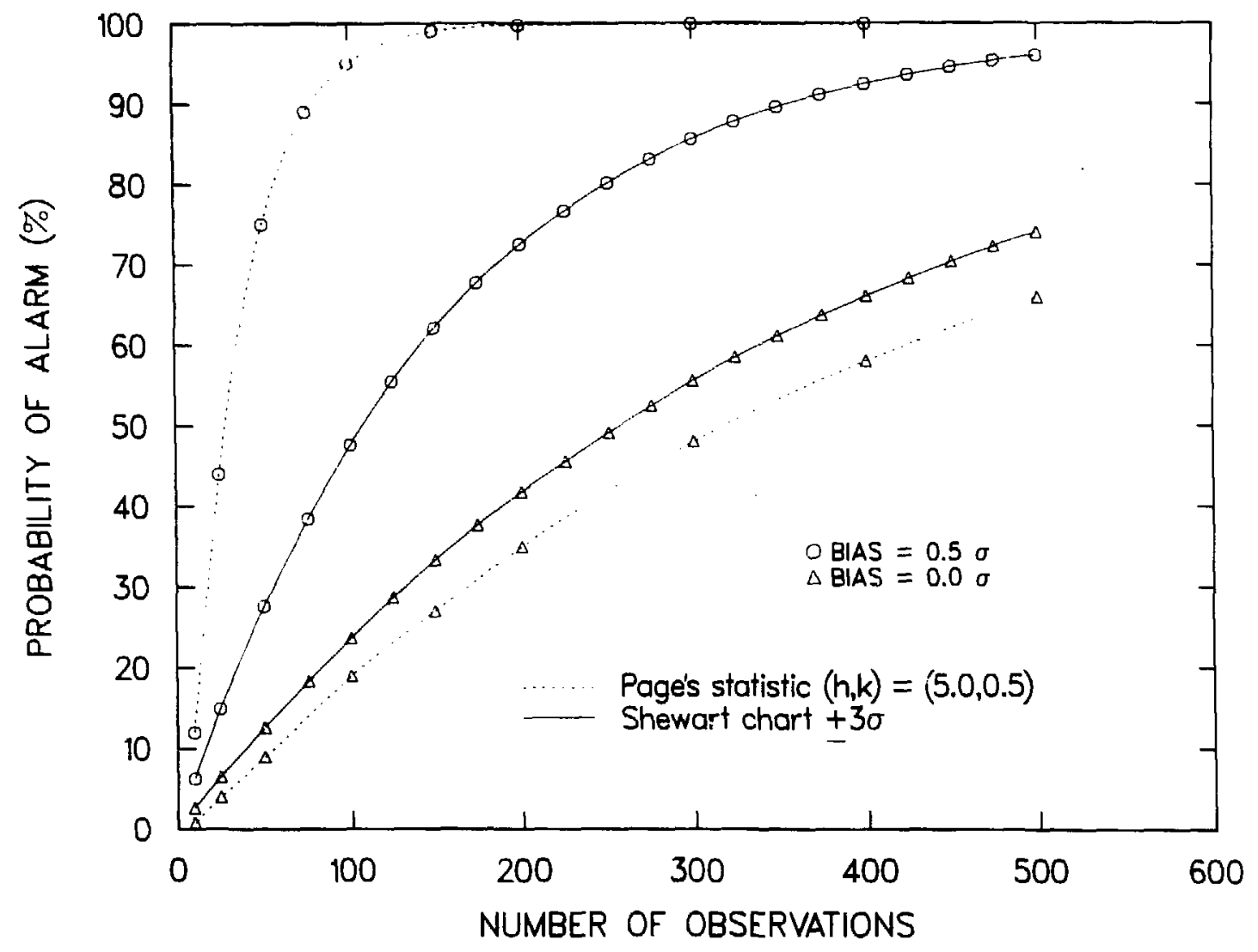

Fig. 2 .

The probability of at least one alarm occurring based on the Page statistic with $(h, k)=(5.0,0.5)$ for standardized measurement biases of 0.0 and 0.5 . Also included is the probability of at least one alarm occurring based on the Shewart chart with control limits at $\pm 3 \sigma$.

The Page statistics $\mathrm{m}(t)$ and $M(t)$ may be plotted against $t$ on a single chart with control limits at $h$ and $-h$. Small changes in bias are reportedly easier to spot on such charts than on standard Shewart control charts.

\section{HODIFICATIONS FOR NONSTANDARD CASES}

The modifications for nonstandard cases consist of replacing the known constant mean $\mu$ and/or known constant standard deviation $\sigma$, used to standardize the observations in Eq. (1), by functions of $t$. Specifically, we 


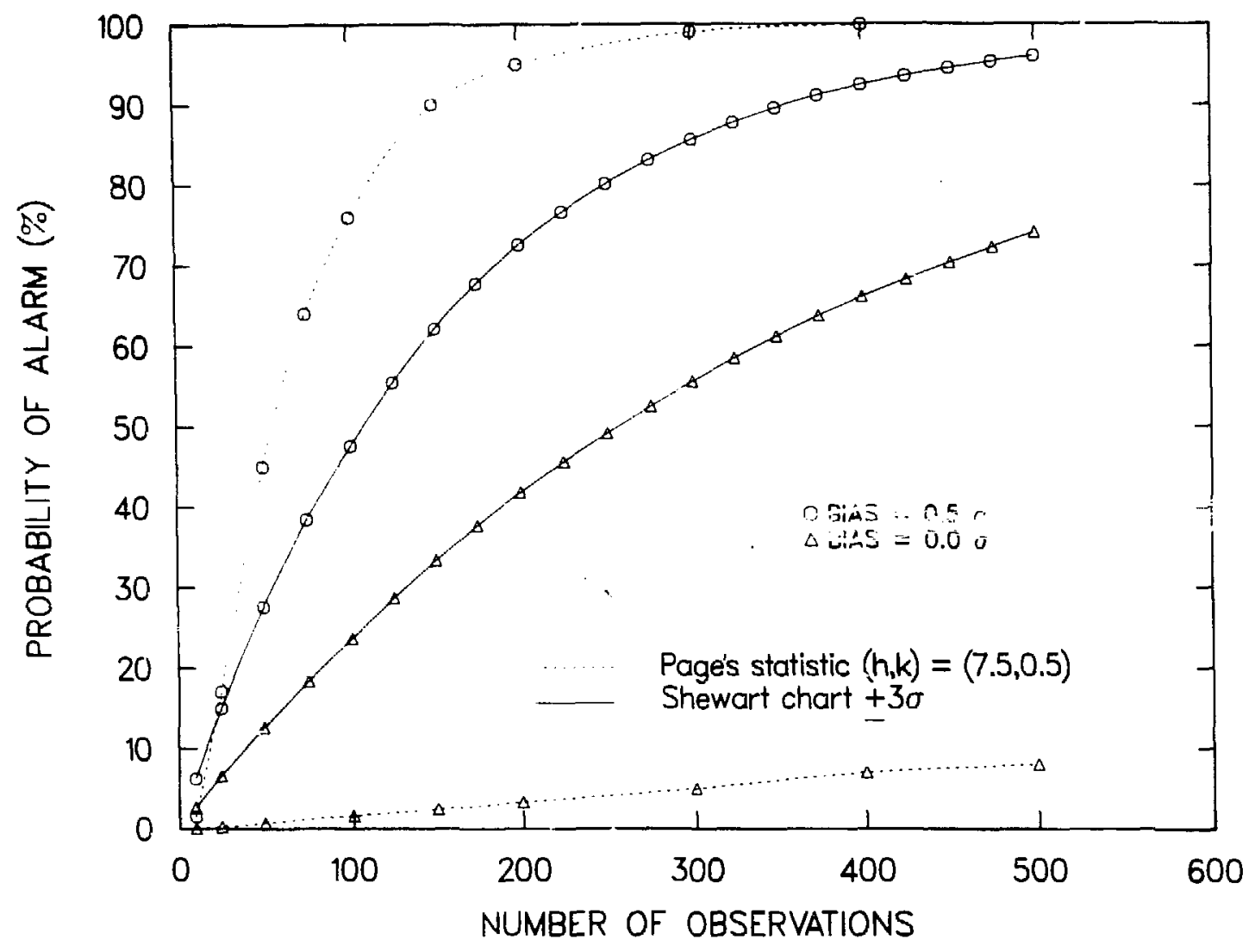

Fig. 3.

The probability of at least one alarm occurring based on the Page statistic with $(h, k)=(7.5,0.5)$ for standardized measurement biases of 0.0 and 0.5 . Also included is the probability of at least one alarm occurring based on the Shewart chart with control limit.s at $\pm 3 \sigma$.

consider the cases where the mean at time $t$ is a nonconstant but known function of $t, \mu_{t}$; where the standard deviation at time $t$ is a nonconstant known or estimated $f$ unction of $t, \sigma_{t}$; and where repeated measurements are made (more than one measurement of the same standard each time a control measurement is scheduled).

\section{A. Modification for Changing (Decaying) Standard Value}

When the standard contains a radioactive element with a relatively short half-life, noticeable changes in the mean value $\mu$ of the measurement process may occur over the usable life of the standard. These changes are easily accommodated if they are not too significant, because the halflife of the element, as well as the initial quantity of material present, 
can be assumed to be known precisely. Hence, the mean value $\mu_{t}$ of the measurement $x_{t}$ at time $t$ can be computed and used to standardize the observation as described in Sec. II.C. Significant decay, however, may also affect the standard deviation $\sigma$, one component of which is a counting error (proportional to the square root of $\mu_{t}$ in a simple Poisson model of the measurement process, but frequently a more complicated function of the counting statistics that takes into account background and internal radioactive sources). One way to compensate for this effect is to increase the counting time used in makin the control measurement. Otherwise, a model for the standard deviation, $\sigma=\sigma_{t}$. in Eq. (1) must be constructed and used as discussed in Sec. III.B.

\section{B. Modification for Deteriorating Measurement Precision}

For nondestructive assay (NDA) instruments with short-lived sources or when the standard itself is decaying, as mentioned in Sec. III.A, one cumponent of the variance may change over time, although the other component. (the part due to factors such as varying atmospheric conditions, sample placement, etc.) remains constant if the measurement process is in control. For this case we reed a model for the changing component or some measurement of the changing component. The constant part is estimated using historical data in a manner similar to that used for the standard case (see Sec. IV.B). The time-dependent standard deviation $\sigma=\sigma_{t}$ needed for standardization of the observation [Eq. (1)] is then computed as the square root of the sum of the computed variance, $\tau_{t}^{2}$, and the constant component, $n^{2}$ :

$$
\sigma_{t}=n^{2}+\tau_{t}^{2}
$$

\section{Modifications When Repeated Measurements Are Made}

Multiple daiiy measurements of the same standard (if, for example, two control measurements are always made at a time) cou1d be treated as independent observations. It is probably more instructive, however, to redefine the control measurement $x_{t}$ as the average of these measurements, 
with the same mean $\mu$ as the original measurement process but with a standard deviation that is smaller by the square root of $n$, the number of replicates. Shifts that can be detected by Page's test using the resulting standardized observations, for example, are similarly smaller by a factor of the juare root of $\mathrm{n}$, with the same parameters as before. (For example, with $=t=i l y$ measurements, we can expect to detect a shift of approximately 0.60 in 12 days while maintaining an average run length of 400 days for the controlled process.)

\section{MAINTENANCE INFORMATION}

The information in this section is not required by the casual user of the measurement control program software but is needed by individuals with responsibility for setting up and maintaining the system. It includes (1) methods for estimating the historical standard deviation needed for standardizing the observations and (2) auxiliary tests of the assumptions -nderlying the control charts and sequential tests (namely, normality and independence of the observations).

\section{A. Estimation of Historical Standard Deviation: Standard Case}

We have assumed that the mean value $\mu$ is known precisely, whether as the result of independent assay or of careful measurement following calibration (in the case of a secondary standard). The value of the standard deviation $\sigma$, however, can be obtained only by observing the process itself over a reasonable length of time (30-60 days, if possible). It depends both on controllable factors, such as standard procedures and the skill of the operator, and on relatively uncontrollable factors, such as air pressure and the inherently random nature of count data. Only some of these factors are captured in the propagated error reported by the software associated with the NDA instrument.

Therefore, to estimate $\sigma$ we require an initial set of observations, which may be obtained during a trial period or, if necessary, during the early phase of actual operation. To the extent possible, these data should 
be collected using procedures, operators, and environments that are typical of actual accountability measurements. (Re-estimation of $\sigma$ may be indicated later, in which case the recent history of the measurement process can be used. However, once computed, $\sigma$, or $\eta$ in the case of the modification described by Eq. (4), is assumed known and constant unless one of the standard tests or control charts indicates that it has been incorrectly estimated or has changed since originally computed.)

Thus, suppose we have a history of $\mathrm{N}$ measurements of the standard. Because these historical data may have some long-term problems (for example, a slow trend or recalibration in the middle of the series), we obtain an estimate of $\sigma$ by segmenting the data into small, more neariy homogeneous subgroups (for example, by weeks); $\sigma^{2}$ is then estimated by the within-group variance of the historical data, rather than by the overal? variance. If the historical data contain some extremely divergent observations (outliers) for which an assignable cause can be determined, these observations should be deleted before performing the computations below, as they can strongly influence the estimate of $\sigma^{2}$. Testing the historical data for normality may also be worthwhile (see Sec. IV.C).

Specifically, then, let the (edited or transformed, if necessary) historical observations be denoted by $x_{i j k}$, where $k=1, \ldots, k$ for $k$ calibration periods, $j=1, \ldots, J_{k}$ for $J_{k}$ subgroups within the $k^{\text {th }}$ calibration period, and $i=1, \ldots, I_{j k}$ for the individual measurements in the $(j, k){ }^{\text {th }}$ subgroup. Form the within-group means:

$$
\bar{x}_{\cdot j k}=\frac{1}{I_{j k}} \sum_{i=1}^{I} x_{i j k}
$$

Then compute

$$
s^{2}=\sum_{k=1}^{K} \sum_{j=1}^{J_{k}} \sum_{i=1}^{I_{j k}}\left(x_{i j k}-\bar{x}_{j j k}\right)^{2} .
$$


Then $\sigma^{2}$ is estimated by

$$
\hat{\sigma}^{2}=\frac{s^{2}}{N-\bar{M}},
$$

where $M$ is the total number of subgroups, that is,

$$
M=\sum_{k=1}^{K} J_{k},
$$

and $\mathrm{N}$ is the total number of observations.

With the data grouped into short intervals, the procedure described in Eqs. (5) through (8) is also suitable for the first nonstandard case considered in Sec. III, namely, a slowly changing mean value $\mu_{t}$, unless $\sigma$ changes significantly over the span of the historical data.

\section{B. Estimation of Historical Standard Deviation: Changing with Time}

Substantial complications are presented by NDA instruments with shortlived sources resulting in measurements of significantly decreasing precision during the periods between replacement. Similar problems occur when a rapidly decaying standard causes large changes in the counting statistics associated with its measurement. We assume that the data available for computation of the historical standard deviation cover a period sufficiently long to reflect such changes and possibly cover at least one replacement of the source (or stanciard). The standard deviation $\sigma_{t}$ to be used in Eq. (1) is computed as the square root of the sum of two variances: first, a component $\tau_{t}^{2}$ associated with the observation at $t i m e t$ and estimated by the software package of the instrument, and second, a constant component $\eta^{2}$ to be estimated from the historical data (given the $\tau_{t}$ 's associated with these data).

We assume that the mean value of the measurement process is approximately constant (or only slowly changing) during the period covered by the 
historical data. (For an instrument with a secondary foil standard, the "known" mean value may, in fact, change when the instrument is recaibrated using primary standards.)

Specifically, let the historical measurements be denoted by $x_{i j k}$ as before, over $K$ calibration periods, with $J_{k}$ groups in the $k^{\text {th }}$ calibration period (grouped observations obviously should not include replacement of the transmission source) and $I_{j k}$ measurements in the $(j, k)^{\text {th }}$ group. Assume that the computed error (that is, the variable component of the measurement variance) is approximately constant in the $(j, k)^{\text {th }}$ group, and denote it by $\tau_{j k}^{2}$. (In practice, $\tau_{j k}^{2}$ is computed as the average of the computed variances of the measurements in the $(j, k)^{\text {th }}$ group.) Compute the withingroup means as in Eq. (5). Then $\eta^{2}$ is estimated by the solving the equation

$$
\sum_{k=1}^{K} \sum_{j=1}^{J_{k}} \sum_{i=1}^{I} \frac{\left(x_{i j k}-\bar{x}_{\bullet j k}\right)^{2}}{n^{2}+\tau_{j k}^{2}}=N-M
$$

numerically. [If $\tau_{\min }^{2}$ and $\tau_{\max }^{2}$ are the smallest and largest values of $\tau_{j k}^{2}$ in the historical data, and if $s^{2}$ is defined by Eq. (6), then the solution to Eq. (9) lies in the range

$$
\left(\frac{s^{2}}{N-M}-\tau_{\max }^{2}, \frac{s^{2}}{N-M}-\tau_{\min }^{2}\right)
$$

and a reasonable starting value for solving Eq. (9) numerically is

$$
\frac{s^{2}}{N-M}-\frac{1}{M} \sum_{k=1}^{K} \sum_{j=1}^{J_{k}} \tau_{j k}^{2}
$$

For a future measurement at time $t$, the expected value of the measurement is $\mu_{t}$, the measured value of the standard obtained immediately after 
the last calibration (or computed in the case of a decaying radioactive standard), and the standard deviation will be $\sigma_{t}$,

$$
\sigma_{t}^{2}=\eta^{2}+\tau_{t}^{2}
$$

where $\tau_{t}^{2}$ is the computed variance returned with that measurement.

\section{Normality and Outliers}

The control charts of Sec. II and other tests are all based on the assumption that measurements obtained from the measurement control program are approximately normally distributed. Failure of normality can affect both the false-alarm rate of the tests and their ability to detect real problems in the process. Therefore we recommend occasionally running a test of normality. Verification of normality of the historical data before estimating the standard deviation that will be used in future control charts and tests is particularly important. If serious deviations from normality are discovered (and if these deviations cannot be ascribed to the presence of a few outliers), we may wish to consider the application of some normalizing transformation to our measurements. For example, distributions with long tails on the right may be made more symmetric by a logarithmic or square root transformation.

The most powerful test of normality found in the literature is the Shapiro-Wilks test. ${ }^{4}$ Application of this test requires a table of coefficients that are different for each sample size and a table of critical values. For practical purposes we set stardard sample sizes $\mathbf{n}$ and storeá the required coefficients $a_{n, i}, i=1,2, \ldots n / 2$, for these sample sizes. Coefficients for samples of size $n=20,30,40$, and 50 are tabulated in Table II. Critical values $c_{n}$ for significance levels (false-alarm rates) of 0.05 and 0.01 are tabulated in Table III. The statistic W for a sample of $n$ observations from the measurement control process is computed as follows :

1. Order the $n$ observations from smallest to largest. Label the ordered observations $x_{1}, \ldots, x_{n}$ (We assume $n$ is even.) 
Table II. Coefficients $a_{n, i}$ for Shapiro-Wilks statistic $w^{a}$

Coefficient

$i$

1

2

3

4

5

6

7

8

9

10

11

12

13

14

15

16

17

18

19

20

21

22

23

24

25
Number of Observations ( $n$ )

\begin{tabular}{llll}
\hline 20 & 30 & 40 & 50 \\
\cline { 2 - 3 } 0.4734 & 0.4254 & 0.3964 & 0.3751 \\
0.3211 & 0.2944 & 0.2737 & 0.2574 \\
0.2565 & 0.2487 & 0.2368 & 0.2260 \\
0.2085 & 0.2148 & 0.2098 & 0.2032 \\
0.1686 & 0.1870 & 0.1878 & 0.1847 \\
0.1334 & 0.1630 & 0.1691 & 0.1691 \\
0.1013 & 0.1415 & 0.1526 & 0.1554 \\
0.0711 & 0.1219 & 0.1376 & 0.1430 \\
0.0422 & 0.1036 & 0.1237 & 0.1317 \\
0.0140 & 0.0862 & 0.1108 & 0.1212 \\
& 0.0697 & 0.0986 & 0.1113 \\
& 0.0537 & 0.0870 & 0.1020 \\
& 0.0381 & 0.0759 & 0.0932 \\
& 0.0227 & 0.0651 & 0.0846 \\
& 0.0076 & 0.0546 & 0.0764 \\
& & 0.0444 & 0.0685 \\
& & 0.0343 & 0.0608 \\
& & 0.0244 & 0.0532 \\
& & 0.0146 & 0.0459 \\
& & 0.0049 & 0.0386 \\
& & & 0.0314 \\
& & & 0.0244 \\
& & & 0.0174 \\
& & & 0.0104 \\
& & & 0.0035
\end{tabular}

$\overline{a_{\text {From Ref. }} \text {, PP. }}$ 603-604.

Table III. Critical values for Shapiro-Wilks testa

Leve1

Number of Observations ( $n$ )

$\begin{array}{lllll}0.01 & 0.868 & 0.900 & 0.919 & 0.930 \\ 0.05 & 0.905 & 0.927 & 0.940 & 0.947\end{array}$

$\overline{\text { a From Ref. 4, p. } 605 . ~}$ 
2. Compute

$$
s^{2}=\sum_{i=1}^{n}\left(x_{i}-\bar{x}\right)^{2},
$$

where $\bar{x}$ is the usual sample mean,

$$
\overline{\mathbf{x}}=\frac{1}{\mathrm{n}} \sum_{i=1}^{\mathrm{n}} \mathrm{x}_{i}
$$

3. Compute

$$
b=\sum_{i=1}^{n / 2} a_{n, i}\left(x_{n-i+1}-x_{i}\right),
$$

where the coefficients $a_{n, i}$ are obtained from Table II.

4. Compute $W=b^{2} / S^{2}$ and compare the result to the critical value with the desired significance level in Table III. If $W$ is smaller than the given critical value, the data appear to depart significantly from normality and should be examined further to delermine if the problem is very light tails, heavy tails, skewness, or perhaps just a smal1 number of outliers for which an assignable cause can be determined.

The storage requirements of this test preclude its routine use for samples of arbitrary size. If, say, 38 historical observations are available, we use the most recent 30 .

A common form of non-normality is a "contaminated normal" distribution, that is, data that are fundamentally normally distributed but that include occasional outliers. When such observations are detected, and when the reason for the change can be determined, these observations should be deleted from the data. 
The most effective way to detect such outliers, given an adequate historical record, is by means of the control chart; points lying outside the action limits are considered outliers. In general, outlier tests look for observations that are substantially removed from the bulk of the data; for example, the tests described by Grubbs ${ }^{5}$ are based on the statistic

$\mathrm{T}_{\text {extreme }}=\frac{\mathbf{x}_{\text {extreme }}-\overline{\mathbf{x}}}{\mathrm{s}}$,

where $x_{\text {extreme }}$ is the observation in the set farthest removed from the sample mean $\bar{x}$ and $s$ is the sample standard deviation. ( $T_{\text {extreme }}$ is called the "extreme studentized residual.") Occasionally it may be useiul to have a test for outiiers that uses only the current week's observations. For detecting a single outlier in samples of size three to eight, the absolute value of $\mathrm{T}_{\text {extreme }}$ above can be compared to the critical values in Table IV, with $x$ extreme being considered an outlier if the critical value is exceeded. This test is not part of MCCAT but may be useful in setting up the system parameters from historical data. Generalizations for the detection of two or more outliers are considered by Rosner. ${ }^{6}$

Table IV. Critical values for absolute value of Textreme

Number of Observations

n

\begin{tabular}{ll}
\multicolumn{2}{c}{ Leve1 } \\
\hline$\underline{0.01}$ & $\underline{0.05}$ \\
1.15 & 1.15 \\
1.49 & 1.46 \\
1.75 & 1.67 \\
1.94 & 1.82 \\
2.10 & 1.94 \\
.22 & 2.03
\end{tabular}

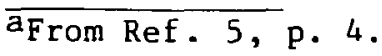




\section{Other Tests of Randomess}

The statement that there is no "assignable cause" contributing to the variability in the measurement control data is equivalent to asserting that the remaining variability is random-the result of uncontrollable factors that vary from day to day or from measurement to measurement. Although randomness is not precisely defined, we have some iniuitive opinions of features that we would not consider :andom, such as extreme observations (four or more standard deviations $\bar{r}$ : oved from the mean) or a slow upward drift in the observations. visible cycles, possibly with weekly or other naturai periods, $c r$ correlations between measurements and other factors, such as the operalor, would alert us to look for potentially controllable ictors affecting the measurements.

A test included in MCCAT that is quite sensitive to many types of non-random fluctuations is based on the von Neumann ratio (the ratio of the mean square successive difference to the variance, or equivalently, the serial correlation coefficient). ${ }^{7,8}$ This test statistic is computed as follows:

1. Let $x_{1}, \ldots, x_{n}$ be $t:$ : $n$ observations in the order in which they were made.

2. Compute

$$
s^{2}=\sum_{i=1}^{n-1}\left(x_{i}-\bar{x}\right)^{2},
$$

where $\bar{x}$ is the usual sample mean.

\section{Compute}

$$
\delta^{2}=\sum_{i=1}^{n-1}\left(x_{i+1}-x_{i}\right)^{2}
$$


4. Compare the value of the ratio $T=\delta^{2} / S^{2}$ with the critical values in Table V. Generally, in measurement control programs, the alternative to no correlation of interest between measurements is the possibility of positive correlation between successive measurements, and so we perform a one-sided test. Positive correlation tends to redice the value of $T$, and thus we would reject the hypothesis of no correlation if $T$ were smaller than the value indicated in Table $\mathrm{V}$.

5. For samples of size $n>25$, compute

$$
z=\left(\frac{n^{2}-1}{n-2}\right)^{1 / 2}\left(\frac{T}{2}-1\right)
$$

Table V. Lower probability points for vor Neumann ratioa

Number of

Observations

n

5

6

7

8

9

10

11

12

13

14

15

16

17

18

19

20

21

22

23

24

25

\begin{tabular}{ll}
\multicolumn{2}{c}{ Level } \\
\hline$\underline{0.01}$ & $\underline{0.05}$ \\
0.538 & 0.820 \\
0.561 & 0.890 \\
0.614 & 0.336 \\
0.665 & 0.982 \\
0.709 & 1.025 \\
0.752 & 1.062 \\
0.791 & 1.096 \\
0.828 & 1.128 \\
0.862 & 1.156 \\
0.893 & 1.182 \\
0.922 & 1.205 \\
0.949 & 1.227 \\
0.974 & 1.247 \\
0.998 & 1.266 \\
1.020 & 1.283 \\
1.041 & 1.300 \\
1.060 & 1.315 \\
1.078 & 1.329 \\
1.096 & 1.342 \\
1.112 & 1.355 \\
1.128 & 1.367
\end{tabular}

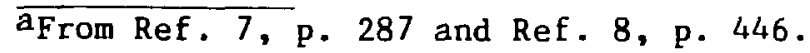


and compare $Z$ with the percentiles of a standard normal. $Z$ is small in the positively correlated case. Thus, the hypothesis of no correlation is rejected at the $5 \%$ level if $Z<-1.645$ or at the $1 \%$ level if $\mathrm{Z}<-2.326$. A two-sided test for either positive or negative correlation between successive measurements rejects the null hypothesis at the $5 \%$ level if $|z|>1.96$ or at the $1 \%$ level iff $|\mathrm{z}|>3.09$.

\section{REFERENCES}

1. S. W. Roberts, "Properties of Control Chart Zone Tests," The Bel1 System Technical Journal 37 , 83-114 (1958).

2. E. S. Fage, "Continuous Inspection Schemes," Biometrika 41, 100-115 (1954).

3. W. D. Ewan and K. K. Kemp, "Sampling Inspection of Continuous Processes with No Autocorrelation Between Successive Results," Biometrika 47, 363-380 (1960).

4. S. S. Shapiro and M. B. Wilk, "An Analysis of Variance Test for Normality (Complete Samples)," Biometrika 52, 591-611 (1965).

5. F. E. Grubbs, "Procedures for Detecting Outlying Observations in Samples," Techncmetrics 11, 1-21 (1969).

6. B. Rosner, "On the Detection of Many Outliers," Technometrics 17, 221227 (1975).

7. C. Bingham and L. S. Nelson, "An Approximation for the Distribution of the von Neumann Ratio," Technometrics 23, 285-288 (1981).

8. B. I. Hart, "Significance Levels for the Ratio of the Mean Square Successive Difference to the Variance," Annals of Mathematical Statistics $12,445-447$ (1942). 